\section{Chronic fatigue syndrome}

Sir: Wessely's review (1997) of books and reports on chronic fatigue syndrome (CFS) fails to mention the ground-breaking report of the independent National Task Force on CFS/PVFS/ME (Tyrrell, 1994). Before producing its report, the Task Force (supported by the Department of Health) consulted widely, held a consensus meeting, and carefully considered the views of the patient organisations. Perhaps for these reasons, its report has escaped the severe criticism, mentioned by Wessely, that met the report of the Royal Medical Colleges (Anonymous, 1996).

Wessely presents a picture of worthy psychiatrists motivated by the "desire for decision-making on the basis of evidence" compared with patients who, driven by emotion, often have "equal and opposite demands". Patients may find this provocative and simplistic, particularly those who, despite the reports of the Task Force and of the Royal Medical Colleges, still find that they are not believed or listened to.

Anomymous (1996) Chronic Fotigue Syndrome: Report of o Committee of the Royal Colleges of Physicions. Psychiatrists and Generol Proctitioners. London: Royal College of Physicians.

Tyrrell, D. (chairman) (1994) Report from the National Tosk Force on Chronic Fatigue Syndrome (CFS). Post Viral Fatigue Syndrome (PVSF), Myalgic Encephalomyelitis (ME). Bristol: Westcare.

Wessely, 5 (1997) Reading about: chronic fatigue syndrome. British fournal of Psychiatry, 171, 92-93.

R. Sykes National Task Force on CFS/PVFS/ME, 155 Whiteladies Road, Clifton, Bristol BS8 2RF

\section{Psychological consequences of road traffic accidents in children and adolescents}

Sir: I read with interest the paper by $\mathrm{Di}$ Gallo et al (1997) about the early psychological consequences of road traffic accidents (RTAs) in children and adolescents, but think their methodology was seriously flawed. The authors included a high proportion of brain-injured subjects (as defined by "lost consciousness") in their cohort of RTA victims. I do not think that the psychological outcome of brain injury can be assumed to be the same as the psychological consequences of "bruises, grazes or lacerations". The confounding effect of so many brain-injured subjects brings into question the conclusions drawn by the authors. In fact, the rate of post-traumatic stress disorder might have been higher if brain-injured subjects had been excluded from the study. I do not think that RTA victims are a homogeneous group and any future research should bear this in mind.

Di Gallo, A., Barton, J. \& Parry-Jones, W. LI. (1997) Road traffic accidents: early psychological consequences in children and adolescents. British Journal of Psychiatry, 170, 358-362.

D. Fearnley Learning Disability Directorate, Ely Hospital, Cambridge Road West, Ely, Cardiff CF5 5XE

Author's reply: I agree with Dr Fearnley that young RTA victims are not a homogeneous group and that the inclusion of subjects with head injury may have influenced the study results. RTAs are frequently associated with head injury, and any consideration of the psychological consequences represents a complex clinical and diagnostic task. Even in the absence of physical signs, or with uncertain ones, head injury can cause a wide range of post-traumatic disturbances. Possible symptoms include anxiety, irritability, sleep disturbances, numbness, memory problems, headaches and dizziness (Alves et al, 1986), all potentially overlapping with symptoms of depression or post-traumatic stress disorder. Often the distinction between definite organic and psychological causation cannot be determined with certainty and, in clinical practice, both need consideration.

Head injury, however, is only one possible confounding factor that has to be considered in analysis and discussion of a study involving RTA victims. There are many others, such as type and circumstances of the accident, or age and developmental stage of the victim. Our study was designed as an exploratory investigation, aimed at describing the early psychological reactions of an unselected sample of child and adolescent RTA victims. In our cohort, psychological symptoms of subjects with head injury did not differ from those without head injury three months following the accident. This result has to be considered in relation to the limitations of methodology and size of our sample. General conclusions can surely not be drawn. I hope that future research will provide more evidence in this neglected field.

Alves, W. M., Coloban, A. R. T., O'Leary, T. J., et al (1986) Understanding post-traumatic symptoms after minor head injury. Journal of Head Traumo Rehabilitation, I, 1-12.

A. Di Gallo Kinder- und jugenpsychiatrische Universitätsklinik und -poliklinik (KJUP).

Schaffhauserrheinweg 55, Postfach CH-4058 Basel, Switzerland
Sleep disturbance and Huntingdon's disease

Sir: It is well recognised that sleep disturbance is a common accompaniment of Alzheimer-type dementia. However, the association between the rare genetic neurodegenerative condition Huntingdon's disease (HD) and sleep problems has received only scant attention. We decided, therefore, to conduct a postal self- or carer-completion questionnaire community survey through the British HD Association.

The response rate was $56.4 \%$ (292 out of 518 ) and the reported prevalence of sleep problems in HD sufferers was $\mathbf{8 7 . 8 \%}$. Sleep problems were rated by $61.7 \%$ as either 'very' or 'moderately' important contributing factors to overall problems. Specifically, the sleep problems reported were, in rank order: restless limb movements, periodic jerky movements, waking during the night, sleepy by day, and early wakening. Perseverance of daytime choreoathetoid movement by night was reported by $50.4 \%$. Over half $(52.7 \%)$ had sought help for their sleep problems, however the sole treatment received (sedative medication) was perceived as being largely unhelpful.

The majority of the principal carers (commonly spouses) also reported significant sleep disruption, primarily attributable to the sufferers' sleep problems, which adversely affected their relationship with the sufferer.

Thus, we conclude that most HD sufferers experience significant sleep problems, which appear to result from one or a combination of the following factors: persistence of abnormal bodily movements, psychiatric comorbidity, poor sleep habits, and sleep fragmentation resulting from neurodegeneration. Given the high rates of sleep disorders and their contribution to the overall burden of illness, it would seem prudent to explore therapeutic strategies within this very needy group.

N. Taylor, D. Bramble Department of Child and Adolescent Psychiatry, University of Nottingham, Queen's Medical Centre, Clifton Boulevard. Nottingham NG7 2UH

\section{Risperidone-induced leucopenia and neutropenia}

Sir: The atypical antipsychotic risperidone is claimed to be haematologically safe (Curtis \& Kerwin, 1995). However, risperidone 